\title{
Chemical Enrichment and the Origin of the Colour-Magnitude Relation of Elliptical Galaxies in a Hierarchical Merger Model
}

\author{
Guinevere Kauffmann ${ }^{1} \&$ Stéphane Charlot ${ }^{2}$ \\ ${ }^{1}$ Max-Planck Institut für Astrophysik, D-85740 Garching, Germany \\ 2 Institut d'Astrophysique du CNRS, 98 bis Boulevard Arago, Paris, France
}

\begin{abstract}
In this paper, we present a model of the formation and chemical enrichment of elliptical galaxies that differs from the conventional picture in two fundamental ways:

1. Ellipticals do not form in a single monolithic collapse and burst of star formation at high redshift. Instead, most of their stars form at modest rates in disk galaxies, which then merge to form the ellipticals.

2. Galaxies do not undergo "closed-box" chemical evolution. Instead, metals can be transferred between the stars, cold gas and the hot gas halos of the galaxies. It is assumed that metals are ejected out of disk galaxies during supernova explosions and these metals enter the hot gas component. The fact that metals are more easily ejected from small galaxies leads to the establishment of a mass-metallicity relation for the disk systems. Big ellipticals are more metal rich because they are formed from the mergers of bigger disks.
\end{abstract}

We use semi-analytic techniques to follow the formation, evolution, and chemical enrichment of cluster elliptical galaxies in a merging hierarchy of dark matter halos. The inclusion of the new metallicity-dependent spectral synthesis models of Bruzual \& Charlot enable us to compute the colours, line indices and mass-to-light ratios of these galaxies. We find that with physically realistic parameters and with the assumption that feedback is efficient, even in massive galaxies, we are able to reproduce the slope and scatter of the colour-magnitude and the $\mathrm{Mg}_{2}-\sigma$ relations. We are not able to match the increase in $M / L$ for bright ellipticals if we assume their mass to measure purely the total quantity of stars in the galaxy. We also study the evolution of these relations to high redshift. We show that the luminosity-metallicity relation does not change with redshift, but the mean stellar age of the galaxies scales with the age of the Universe. This is why the evolution of cluster ellipticals appears to be well described by simple passive evolution. Finally, we study the enrichment history of the intracluster gas. Our models predict that more than $80 \%$ of the metals were ejected by galaxies with circular velocities less than $250 \mathrm{~km} \mathrm{~s}^{-1}$ at redshifts greater than 1 . The metallicity of the ICM is thus predicted to evolve very little out to $z>1$.

Keywords: galaxies:formation,evolution; galaxies: elliptical and lenticular; galaxies: stellar content 


\section{Introduction}

The relationship between the colours, line strengths and luminosities of elliptical galaxies is potentially a source of information about physical processes important during their formation. The conventional interpretation of the colour-magnitude relation of ellipticals is that it is primarily a metallicity effect (Faber 1977; Dressler 1984; Vader 1986). It is often assumed that elliptical galaxies form monolithically in a single giant burst of star formation at high redshift. Supernova explosions heat the gas, and when its thermal energy exceeds its binding energy, a galactic wind is produced, which is assumed to interrupt further star formation and enrichment. Since the binding energy per unit mass of gas is higher in more massive galaxies, these are able to retain their gas for much longer times and so to reach higher metallicities than less massive galaxies (Larson 1974; Matteuci \& Tornambé 1987; Arimoto \& Yoshii 1987; Bressan et al 1996). This interpretation is not unique, however, because colours and line strengths are degenerate in age and metallicity. As a result, the colour-magnitude relation of local ellipticals can also be interpreted as an increase in mean stellar age with luminosity (Worthey 1996). In recent work, Kodama \& Arimoto (1997) have pointed out that the evolution of the colour-magnitude relation to high redshift does not support the hypothesis that age effects alone are responsible for the redder colours of the brighter ellipticals. Following this, Charlot, Ferreras \& Silk (1997, in preparation) have explored models where both age and metallicity are important, and have shown that several of these can provide equally good fits to the data.

An alternative approach to interpreting the spectra of ellipticals is to begin with a more realistic dynamical picture for the formation of these objects, and then to study the properties of their stellar populations by applying spectral synthesis techniques. Recently, there has been considerable progress in modelling the observed properties of ellipticals in a scenario where they are formed by the merging of disk galaxies in a universe where structure is built through hierarchical clustering (Kauffmann 1996b, Baugh, Cole \& Frenk 1996). This work has, for the first time, placed the theory of the formation and evolution of elliptical galaxies in a proper cosmological context. In these models, star formation takes place mainly in centrifugallysupported disks, formed when gas cools and condenses at the centres of virialized halos of dark matter. A self-regulated balance between cooling and feedback from supernova explosions maintains star formation at a roughly constant rate in these disks. If two disk galaxies merge once their surrounding halos have coalesced, an elliptical galaxy is formed and all cold gas present in the merger remnant is transformed instantaneously into stars in a "burst". Kauffmann (1996b) showed that although ellipticals are predicted to have been assembled by relatively recent mergers $(z<1)$ in a standard cold dark matter (CDM) cosmology, most of their stars form at considerably higher redshift in their disk-galaxy progenitors. The $V$-band luminosity-weighted stellar age of cluster ellipticals was shown to vary between 8 and 12 Gyears and the scatter in the predicted $V-K$ and $U-V$ colours was of order 0.04 , consistent with the small observed scatter of Virgo and Coma cluster elliptical colours (Bower, Lucey \& Ellis 1992). The model presented in that paper did not include any scheme for the chemical enrichment of galaxies and it was unable to reproduce the slope of the observed colour-magnitude relation. An important conclusion of this work was that age effects in hierarchical models could not alone account for the redder colours of the more massive ellipticals.

We have now included a simple prescription for chemical evolution in our semi-analytic 
galaxy formation scheme in order to analyze the effect of variations in metallicity on the observed properties of ellipticals. Supernovae take place in stars forming disk galaxies and the explosions cause cold gas and metals to be ejected into the halo, where the metals are mixed with the hot halo gas. The efficiency with which this process occurs depends on the potential well depth, so that less massive galaxies eject more material and hence produce fewer stars and metals per unit mass of available cold gas. The metallicity of disk galaxies thus increases as a function of their mass. We then show that in a hierarchical merger picture, bright ellipticals form from mergers of substantially more massive disks than faint ellipticals. This lays the theoretical groundwork for understanding the origin of a mass-metallicity relation for elliptical galaxies.

We then compare the predictions of the model with observational data. To do this, we make use of the new stellar population synthesis models of Bruzual \& Charlot (1997, in preparation); these enable us to compute the colours and spectral line indices of evolving stellar populations of different metallicities. We show that with physically reasonable choices for the free parameters in our model, we are able to reproduce both the slope and the scatter of the $U-V$ and $V-K$ colour-magnitude relations of cluster ellipticals. In addition, we obtain a good fit to the slope of observed relation between the $\mathrm{Mg}_{2}$ index and the stellar velocity dispersion $\sigma$, where it is assumed that $\sigma$ simply scales in proportion to the circular velocity of the halo in which the elliptical galaxy formed. We also examine the evolution of these relations to high redshift and show that out to a redshift of 1 in a standard CDM cosmology, the slopes change very little and the colours and index values at fixed luminosity shift to lower values as expected for a passively evolving population of stars. At redshifts greater than one, there is a slight flattening in the slopes of the relations, caused by the slightly younger mean ages of the bright ellipticals relative to the faint ones.

Although the colour-magnitude relations are reproduced very well by our model, we can-

not account for the observed increase in the mass-to-light ratios of bright elliptical galaxies if we assume the mass, estimated using the central line-of-sight velocity dispersion $\sigma$, is purely a measure of the total quantity of stars in the galaxy. We hypothesize that since massive ellipticals are built up through a greater number of mergers than their less massive counterparts, more dark matter may end up being mixed with their stars. Finally, since our chemical evolution model tracks the recycling of heavy elements between stars, cold gas, and the hot intergalactic medium, we are able to study the enrichment history of the hot gas in clusters. With a Sn Ia + Sn II yield of twice solar, we are able to reproduce the observed ratio of iron mass to total blue luminosity in nearby clusters (Renzini et al 1993). We show that $80 \%$ of the metals are produced at redshifts greater than 1 in galaxies with circular velocities less than $250 \mathrm{~km} \mathrm{~s}^{-1}$. The metallicity of rich clusters is predicted to evolve very little out to redshifts of 1 and beyond.

\section{Modelling the Formation and Chemical Enrichment of Elliptical Galaxies}




\subsection{Semi-analytic models of elliptical galaxy formation in a hierar- chical Universe}

The semi-analytic model we employ is the same as that used by Kauffmann (1996b) to compute the stellar ages of elliptical galaxies and bulges formed by mergers in a cold dark matter universe. The reader is referred to that paper for more background. The new material in the present paper is a prescription for chemical enrichment and the transfer of metals between stars, cold galactic gas and hot halo gas. We describe this in detail in the next section. More details about semi-analytic techniques may be found in Kauffmann \& White (1993), Kauffmann, White \& Guiderdoni (1993, KWG), Cole et al (1994) and Baugh, Cole \& Frenk (1996).

To summarize:

An algorithm based on an extension of the Press-Schechter theory due to Bower (1991) and to Bond et al (1991) is used to generate Monte Carlo realizations of the merging paths of dark matter halos from high redshift until the present. This algorithm allows all the progenitors of a present-day object, such as a cluster, to be traced back to arbitrarily early times. Dark matter halos are modelled as truncated isothermal spheres and it is assumed that as the halo forms, the gas relaxes to a distribution that exactly parallels that of the dark matter. Gas then cools and condenses onto a central galaxy at the core of each halo. Star formation and feedback processes take place as described in KWG (see also below). In practice, star formation in central galaxies takes place at a roughly constant rate of a few solar masses per year, in agreement with the rates derived by Kennicutt (1983) for normal spiral galaxies.

As time proceeds, a halo will merge with a number of others, forming a new halo of larger mass. All gas which has not already cooled is assumed to be shock heated to the virial temperature of this new halo. This hot gas then cools onto the central galaxy of the new halo, which is identified with the central galaxy of its largest progenitor. The central galaxies of the other progenitors become satellite galaxies, which are able to merge with the central galaxy on a dynamical friction timescale. If a merger takes place between two galaxies of roughly comparable mass, the merger remnant is labelled as an "elliptical" and all cold gas is transformed instantaneously into stars in a "starburst". Note that the infall of new gas onto satellite galaxies is not allowed, and star formation will continue in such objects only until their existing cold gas reservoirs are exhausted. Thus the epoch at which a galaxy is accreted by a larger halo delineates the transition between active star formation in the galaxy and passive evolution of its stellar population. The stellar populations of elliptical merger remnants in clusters hence redden as their stellar populations age. Central galaxy merger remnants in the "field" are able to accrete new gas in the form of a disk to form a "spiral" galaxy consisting of both a spheroidal bulge and a disk component. Note that as in KWG, we suppress the formation of visible stars in cooling flows within halos with $V_{c}>500 \mathrm{~km} \mathrm{~s}^{-1}$ in order to avoid producing central cluster galaxies that are too bright and too blue.

The cosmological initial conditions used in this paper are a $\sigma_{8}=0.67$ CDM universe with $\Omega=1$ and $H_{0}=50 \mathrm{~km} \mathrm{~s}^{-1} \mathrm{Mpc}^{-1}$. Although this model does not match the COBE measurements of the amplitude of the microwave background fluctuations, it has become the de facto standard for galaxy formation studies, since it provides a reasonable fit to observed small-scale galaxy clustering. 


\subsection{The chemical enrichment of galaxies in a hierarchical Universe}

In simple closed-box models of chemical evolution, all metals produced by stars are retained within the galaxy. After all the gas has been converted into stars, the metallicity of the galaxy is then simply given by the mass of metals produced per solar mass of long-lived stars that are formed. In our model, metals are exchanged between three components: stars, cold galactic gas, and hot halo gas. The routes through which this exchange may take place are the following:

1. Stars $\longrightarrow$ hot and cold gas As decribed in KWG, the star formation rate in disk galaxies is given by

$$
\dot{M}_{\text {stars }}=\alpha M_{\text {cold }} / t_{d y n},
$$

where $M_{\text {cold }}$ is the mass of cold gas in the galaxy, $t_{d y n}=R_{v i r} / 10 V_{c}$ is a measure of the dynamical time of the galaxy (this assumes that the galaxy collapses by a factor 10 from the virial radius of the halo), and $\alpha$ is a free parameter. We assume that stars form with a standard Scalo (1986) IMF truncated at 0.1 and $100 M_{\odot}$ and use the instantaneous recycling approximation (e.g. Tinsley 1980) to compute the amount of gas and metals returned to the interstellar medium and halo. A yield $y$ of heavy elements is produced per solar mass of gas converted into stars. A fraction $f$ of this yield is uniformly mixed with the hot gas in the halo, and the rest is uniformly mixed with the cold gas in the galaxy. The parameter $f$ is introduced to allow for the possibility that heavy elements may be ejected directly into the halo in galactic fountains or chimneys resulting from the simultaneous explosions of tens or hundreds of supernovae (see Spitzer 1990 for a review). The gas fraction returned by evolved stars is estimated from the population synthesis models to be $25 \%$ and is roughly independent of metallicity. This material is added to the cold gas component of the galaxy.

2. Cold gas $\longrightarrow$ hot gas In our models, it is postulated that supernova explosions can release enough energy to drive cold gas into the halo. For a standard Scalo IMF, the number of supernovae expected per solar mass of stars formed is $\eta_{S N} \simeq 5 \times 10^{-3} M_{\odot}^{-1}$. The kinetic energy of the ejecta from each supernova is $10^{51}$ ergs. If a fraction $\epsilon$ of this energy is used to reheat cold gas to the virial temperature of the halo, the amount of cold gas lost to the intergalactic medium in time $\Delta t$ may be estimated from simple energy balance arguments as

$$
\Delta M_{\text {reheat }}=\epsilon \frac{4}{5} \frac{\dot{M}_{*} \eta_{S N} E_{S N}}{V_{c}^{2}} \Delta t
$$

Here $\epsilon$ is a free parameter controlling the efficiency of the feedback process. When cold gas is returned to the halo, it is assumed that its metals are uniformly mixed with the halo gas.

3. Hot gas $\longrightarrow$ cold gas Metals present in hot halo gas may incorporated into a galaxy when that gas is able to cool and condense at the centres of halos. The cooling rates in halos are calculated as described in KWG and are based on the model by White \& Frenk (1991). The cooling functions vary according to the metallicity of the hot gas and are interpolated from Figure 9.9 of Binney \& Tremaine (1987). 
4. Mixing during halo mergers As structure in the Universe builds up through hierarchical clustering, dark matter halos merge and the metals contained in their hot gas components are mixed together. In this way, metals ejected by star-forming galaxies in the "field" at high redshift may end up contributing to the metals in the gas in rich clusters at $z=0$.

5. Mixing during galaxy mergers When two galaxies merge within a common dark matter halo, the metals contained in the cold gas and stars of both objects will be mixed in the merger remnant. We divide galaxy-galaxy merging events into two different classes: a) accretion events - this is when one galaxy is less than a third of the mass of the other one. b) major mergers - the galaxies are within a factor of three in mass. Major mergers are assumed to lead to the formation of elliptical galaxies. All the cold gas contained in the galaxies is turned into stars instantaneously in a "burst" and the enrichment during this phase is assumed to be a closed-box process, i.e. no metals escape from the galaxy. Accretion events do not induce any extra star formation. The metals present in the cold gas and stellar component of the satellite are simply added to the metals in the cold gas and stars of the central galaxy. Note that the factor of three is chosen so that the central galaxies of halos of circular velocity $\sim 200 \mathrm{~km} \mathrm{~s}^{-1}$ will, on average, have bulge-to-disk ratios consistent with an Sbc-type spiral galaxy (see KWG). Our rules about star formation and enrichment during mergers and accretion events have been chosen for the sake of simplicity. They do not affect the results presented in this paper because, as we have mentioned, most stars in ellipticals do not form in these phases.

To summarize, the main parameters of our model that control the metallicities of the stellar, cold gas and hot gas components in galaxies are the following:

- $\Omega_{b}$ the density of the Universe in baryons

- $\alpha$ the star formation efficiency

- $\epsilon$ the feedback efficiency

- $y$ the yield of heavy elements (instantaneous recycling assumed)

- $f$ the fraction of heavy elements ejected directly into the hot halo gas without first being mixed with the cold gas

As in previous work (see KWG), we have adopted a "Milky Way" normalization, i.e. we tune the parameters so that the properties of the central galaxy in a halo of $V_{c}=220 \mathrm{~km} \mathrm{~s}^{-1}$ match those of our own Galaxy. In practice, the tuning procedure operates as follows. We first choose a value of $\Omega_{b}$. $\alpha$ and $\epsilon$ control the luminosity and cold gas mass of the galaxy and are adjusted to obtain a $B$-band absolute magnitude of $\sim-20.5$ and a cold gas mass of $6 \times 10^{9} M_{\odot}$. The yield is chosen so that the galaxy has a $V$-light weighted mean metallicity of 0.7 solar, a value consistent with the mean observed metallicity of stars in the solar neighbourhood (see Tinsley 1980 and references therein). It should be noted that in our model, since metals are driven out of a galaxy by winds and diluted in the surrounding gas, the mean metallicity of the stars in a galaxy will always be lower than the yield $y$. The higher the feedback efficiency, the higher the value of $y$ required to obtain a metallicity of 0.7 solar. The parameter $f$ controls the 
total mass of metals contained in the hot halo gas. Since there are no reliable measurements of the metallicities of galactic halo gas (indeed, X-ray emission from hot gas around spiral galaxies has yet to be detected), we tune $f$ to match the abundance of metals observed in the intracluster medium. A compilation of data by Arnaud et al (1992) shows that the Fe abundance in a sample of nearby clusters is $\sim 0.3$ solar, with substantial scatter. We have thus chosen $f$ so that the metallicity of the hot gas in halos of circular velocity $1000 \mathrm{~km} \mathrm{~s}^{-1}$ matches this value. Note that the properties of a Milky Way-type disk galaxies were explored in detail in a paper by Kauffmann (1996a) using a chemical evolution model very similar to the one described above. In that paper, it was demonstrated that the classic G-dwarf problem was solved by rapid early enrichment in the low mass objects that later merged to form the Galaxy, and that the predicted age-metallicity distribution of the stars in the Galactic disk agreed rather well with a recent compilation of data on solar neighbourhood stars.

In Table 1, we list parameter values for two representative models. Model A (or the strong feedback model) has $\Omega_{b}=0.1$. For our adopted Hubble Constant of $50 \mathrm{~km} \mathrm{~s}^{-1} \mathrm{Mpc}^{-1}$, this value lies at the upper end of the permitted range of $\Omega_{b}$ according to current constraints from models of Big Bang nucleosynthesis (see Turner 1996 for a review), but at the lower end of measurements the fraction of baryons in rich clusters (White et al 1993; White \& Fabian 1995). Model A also has a high supernova feedback efficiency. The value of the yield (twice solar) is rather high, but still roughly consistent with theoretical calculations of the mass of different types of heavy elements ejected during Type II and Type Ia supernova explosions assuming a standard Salpeter IMF (Tsujimoto et al 1995 ). Model B (or the weak feedback model) has $\Omega_{b}=0.06$. It has a considerably smaller feedback efficiency and yield.

\section{The New Spectral Synthesis Models}

We compute the spectrophotometric properties of model galaxies using new population synthesis models by Bruzual \& Charlot (1997, in preparation). These span the range of metallicities $5 \times 10^{-3} \leq Z / Z_{\odot} \leq 5$ and include all phases of stellar evolution from the zero-age main sequence to supernova explosions for progenitors more massive than $8 M_{\odot}$, or the end of the white dwarf cooling sequence for less massive progenitors. The models are based on recent stellar evolutionary tracks computed by Alongi et al. (1993), Bressan et al. (1993), Fagotto et al. (1994a, b, c), and Girardi et al. (1996), supplemented with prescriptions for upper-AGB and post-AGB evolution. The radiative opacities are taken from Iglesias et al. (1992). In the version used here, we adopt the library of synthetic stellar spectra compiled by Lejeune et al. (1997b, in preparation; see also 1997a) for all metallicities. This library is based on spectra by Kurucz (1995, private communication; see also Kurucz 1992) for the hotter (O-K) stars, Bessell et al. (1989, 1991) and Fluks et al. (1994) for M giants, and Allard \& Hauschildt (1995) for M dwarfs. The Lejeune et al. spectral library also includes semi-empirical corrections for blanketing, a well-known limitation of synthetic spectra (see, for example, Charlot, Worthey \& Bressan 1996, and references therein). The resulting model spectra computed for stellar populations of various ages and metallicities have been checked against observed spectra of star clusters and galaxies (Bruzual \& Charlot 1997; Bruzual et al. 1997).

In addition, the models predict the strengths of 21 stellar absorption features, including the $\mathrm{Mg}_{2}$ index near $5000 \AA$ that is often used as a spectral diagnostic in early-type galaxies. 
The predictions are computed using the Worthey et al. (1994) analytic fitting functions for index strength as a function of stellar temperature, gravity and metallicity that are calibrated empirically on a sample of 460 Galactic stars. This constitutes the standard "Lick/IDS" system, in which each index is defined by a central bandpass bracketted by two pseudocontinuum bandpasses. The integrated index of a stellar population is then computed by weighting the contributions from individual stars by their level of continuum (see Worthey et al. 1994, and Bressan et al. 1996 for more detail). It is worth noting that the most massive elliptical galaxies exhibit $[\mathrm{Mg} / \mathrm{Fe}]$ ratios in excess of that found in the most metal-rich stars in the solar neighborhood (by $\sim 0.2-0.3$ dex; see Worthey, Faber, \& Gonzalez 1992). While this may limit the accuracy of the predicted $\mathrm{Mg}_{2}$ indices of bright elliptical galaxies, the recent models of Bressan et al. (1997, in preparation) show convincingly that an enhancement in light elements at fixed total metallicity has virtually no effect on the other spectrophotometric properties of model stellar populations.

A complete discussion of the differences between the spectrophotometric predictions of these models with those in previous studies will be presented in Bruzual \& Charlot (1997). The typical discrepancies between the properties of stellar populations of the same input age and metallicity that are obtained by using the spectral synthesis models constructed by different groups of scientists, have already been illustrated by Charlot, Worthey \& Bressan (1996). These can reach up to $0.05 \mathrm{mag}$ in rest-frame $B-V, 0.25 \mathrm{mag}$ in rest-frame $V-K$ and a $25 \%$ dispersion in the $V$-band mass-to-light ratio. With these uncertainties in mind, we will concentrate more on understanding the trends seen in the observations than on obtaining exact fits to the data.

\section{Theory of the origin of a mass-metallicity relation for ellipticals}

As was discussed previously, the assumption that supernova feedback ejects cold gas and metals from galaxies and that the efficiency of this process scales as $1 / V_{c}^{2}$, where $V_{c}$ is the circular velocity of the galaxy, means that more massive and luminous disk galaxies will be more metal rich. Because of the $1 / V_{c}^{2}$ scaling, the metallicity-luminosity relation will saturate at large $V_{c}$ when the feedback efficiency becomes negligible and all gas and metals are retained in the galaxy. For large $V_{c}$, galaxies are effectively closed boxes. Thus in our scenario, metals in the hot gas component, including the gas in rich clusters, are produced primarily by lowmass galaxies since these lose their gas very easily. In clusters, most of the enrichment takes place at high redshift before the cluster has assembled (see section 8).

In figure 1, we show the metallicity-luminosity relation for disk galaxies at $z=0$ in our model. We have plotted the $V$-light weighted mean metallicities of the stars in these disks. The result for model $\mathrm{A}$ is shown as a solid line and that for $\mathrm{B}$ as a dashed line. As expected the relation rises much more steeply for model A, which has much more efficient feedback. In model $\mathrm{B}$, the relation saturates at $V$-absolute magnitudes of around -20 . The model $\mathrm{A}$ relation is in better qualitative agreement with the observed metallicity-luminosity relation of spiral galaxies (measured using emission lines from the gas in HII regions) since this shows no turnover (Roberts \& Haynes 1994).

In the merger model, it is not a priori clear that ellipticals should also obey a metallicity- 


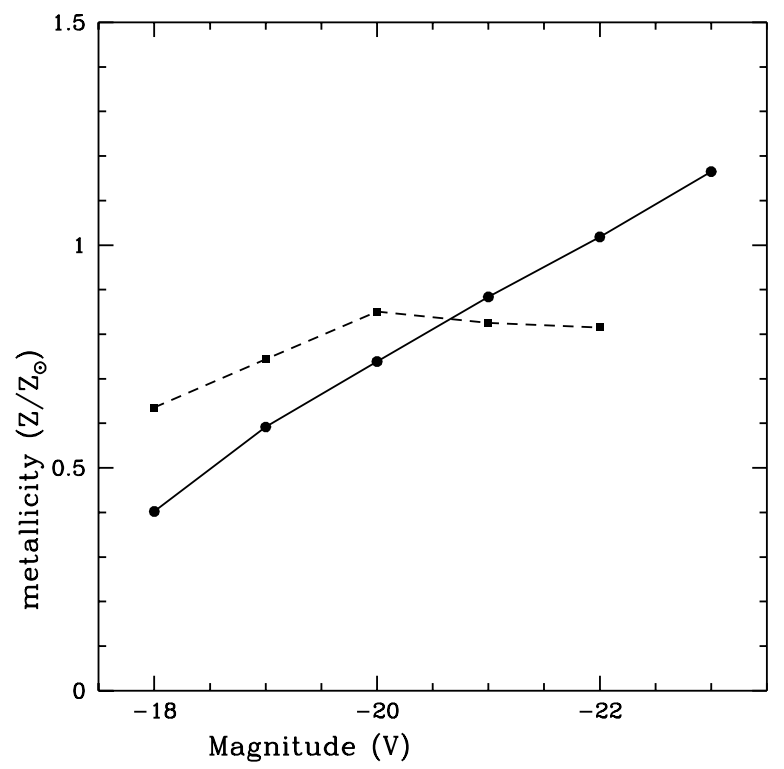

Figure 1: The metallicity-luminosity relation for disk galaxies in the model: the $V$-luminosity weighted mean metallicity of the stars is plotted as a function of $V$ - band absolute magnitude. The solid line is for the high feedback model A and the dashed line is for the low feedback model B.

luminosity relation. For this to be the case, bright ellipticals have to be formed from the mergers of systematically bigger disks than faint ellipticals. Figure 2 demonstrates that this is indeed the case. We plot the mean progenitor mass of ellipticals in present-day clusters of circular velocity $1000 \mathrm{~km} \mathrm{~s}^{-1}$ versus their stellar mass. The mean progenitor mass is computed by weighting each star in the galaxy by the mass of the disk-galaxy progenitor in which it formed and is expressed in units of the total mass of the elliptical. As can be seen, the progenitors of elliptical galaxies of $2 \times 10^{11} M_{\odot}$ are typically 10 times more massive than those of $10^{10} M_{\odot}$ ellipticals. The error bars show the 5 th to 95 th percentile spread in mean progenitor mass among ellipticals in each mass bin.

In figure 3 , we show the metallicity-luminosity and age-luminosity relations for presentday cluster ellipticals. The left panel is for model A and the right panel is for model B. We plot $V$-light weighted stellar quantities as before. Note that the ages differ somewhat from those given in Kauffmann (1996b), because the $V$-light weighting has changed as a result of the implementation of the new spectral synthesis models. The rms scatter in age is 1.2 Gyear and the scatter in metallicity at fixed luminosity is 0.14 in solar units. Model A again has a much steeper metallicity-luminosity relation than model B. Both models show very little trend in age with luminosity. If anything, the brighter ellipticals have somewhat younger ages. We conclude, therefore, that in the hierarchical merger model, the observed colour-magnitude relation for elliptical galaxies must arise as a metallicity effect. 


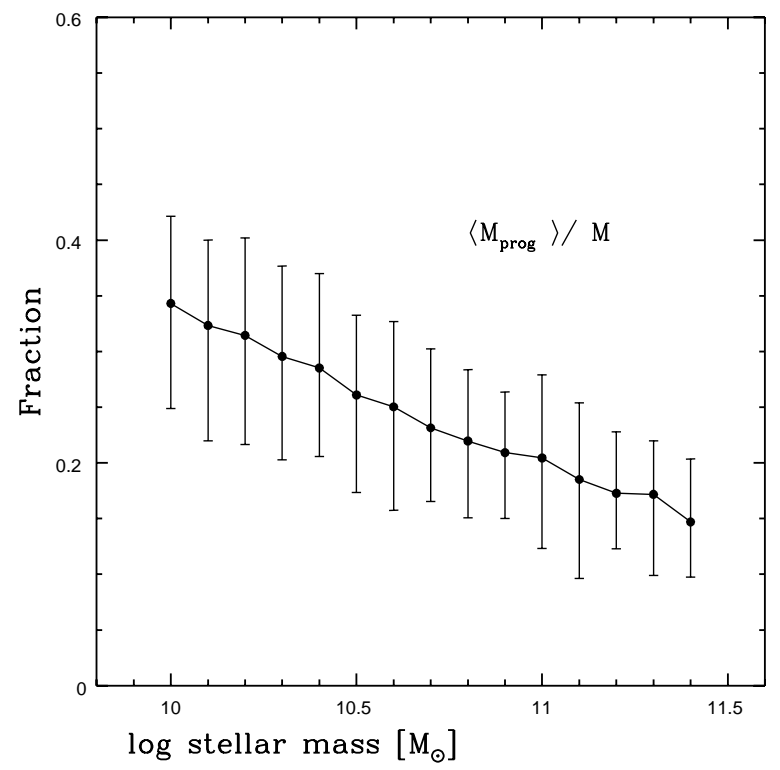

Figure 2: The mean progenitor mass of ellipticals in clusters of circular velocity $1000 \mathrm{~km} \mathrm{~s}^{-1}$ is plotted as a function of their stellar mass. The progenitor mass is expressed as a fraction of the final mass of the elliptical. Errorbars show the 5th-95th percentile range in progenitor mass.

\section{The colour-magnitude and $\mathrm{Mg}_{2}-\sigma$ relation for cluster ellipticals}

In figure 4, we show the $U-V$ and $V-K$ colour-magnitude relations for cluster ellipticals. Results for model A are shown in the left-hand panel and results for model $\mathrm{B}$ in the right. The straight lines are the fits to the $U-V$ and $V-K$ colour-magnitude relations of elliptical galaxies in the Virgo and Coma clusters derived by Bower, Lucey \& Ellis (1992, hereafter BLE). Model A fits the observations very well. We have computed least-squares estimates of the slopes and scatter of the Model A relations and compare them in Table 2 with the values derived by BLE for the Coma and Virgo ellipticals. Note that the galaxies plotted in figure 4 are from 5 different Monte Carlo realizations of the formation of a cluster of $V_{c}=1000 \mathrm{~km}$ $\mathrm{s}^{-1}$. Even so, the relation has very small scatter. Since model A fits the data so much better than model $\mathrm{B}$, in future we show results only for model A.

It is well known that the $\mathrm{Mg}_{2}$ line index correlates strongly with the stellar velocity dispersion of elliptical galaxies (e.g. Bender, Burstein \& Faber 1993). In our models, this relation is less straightforward to derive as we do not have a dynamical model for computing $\sigma$. For simplicity, we have assumed a constant ratio $V_{c} / \sigma$, where $V_{c}$ is the circular velocity of the halo in which the elliptical formed during its last major merger. If the stars were a non-selfgravitating test population with density profile $\propto r^{-3}$ within a singular isothermal halo, one would have $V_{c} / \sigma=3^{1 / 2}$ (Gunn 1982).

The model $\mathrm{Mg}_{2}$ indices, computed using the population synthesis models as described in 


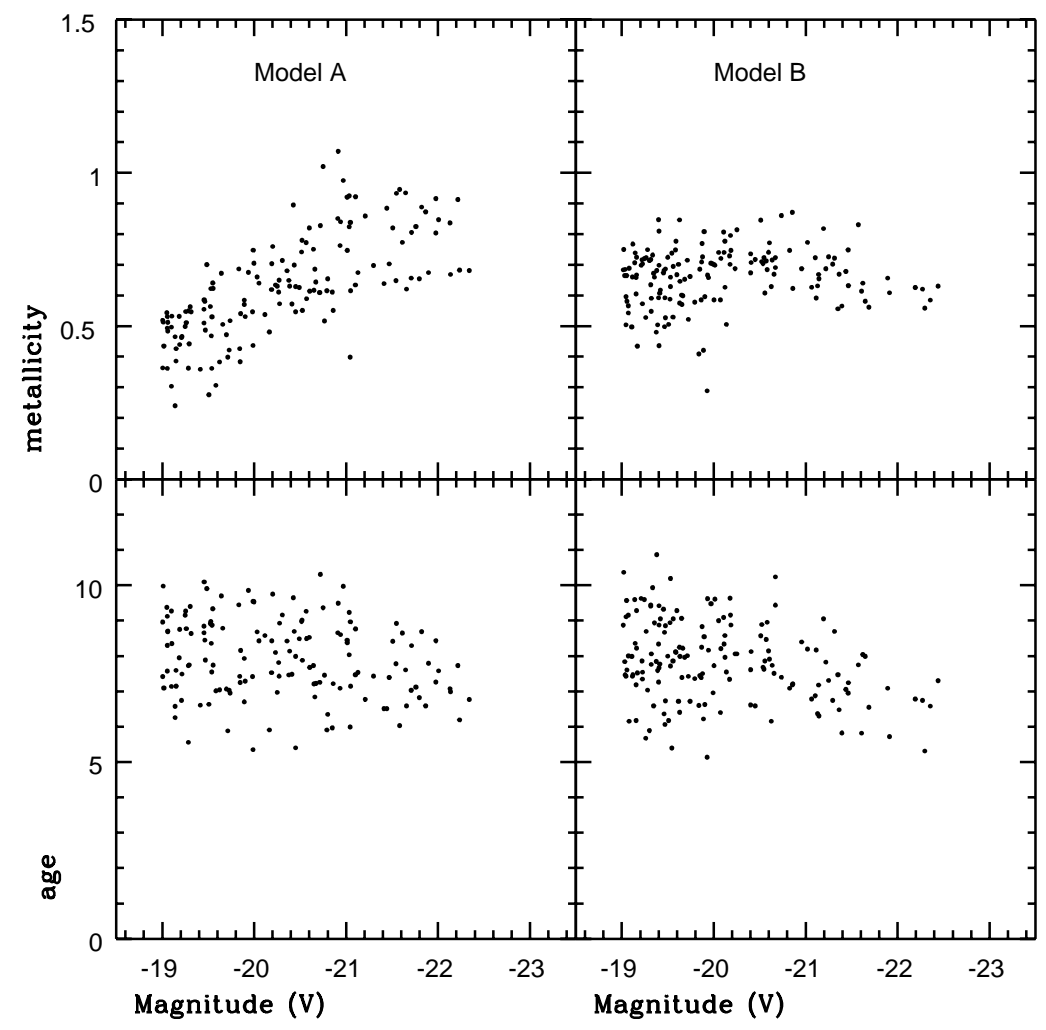

Figure 3: The metallicity-luminosity and age-luminosity relations of elliptical galaxies in clusters of circular velocity $1000 \mathrm{~km} \mathrm{~s}^{-1}$ at the present day . $V$-luminosity weighted quantities are plotted, as before. 


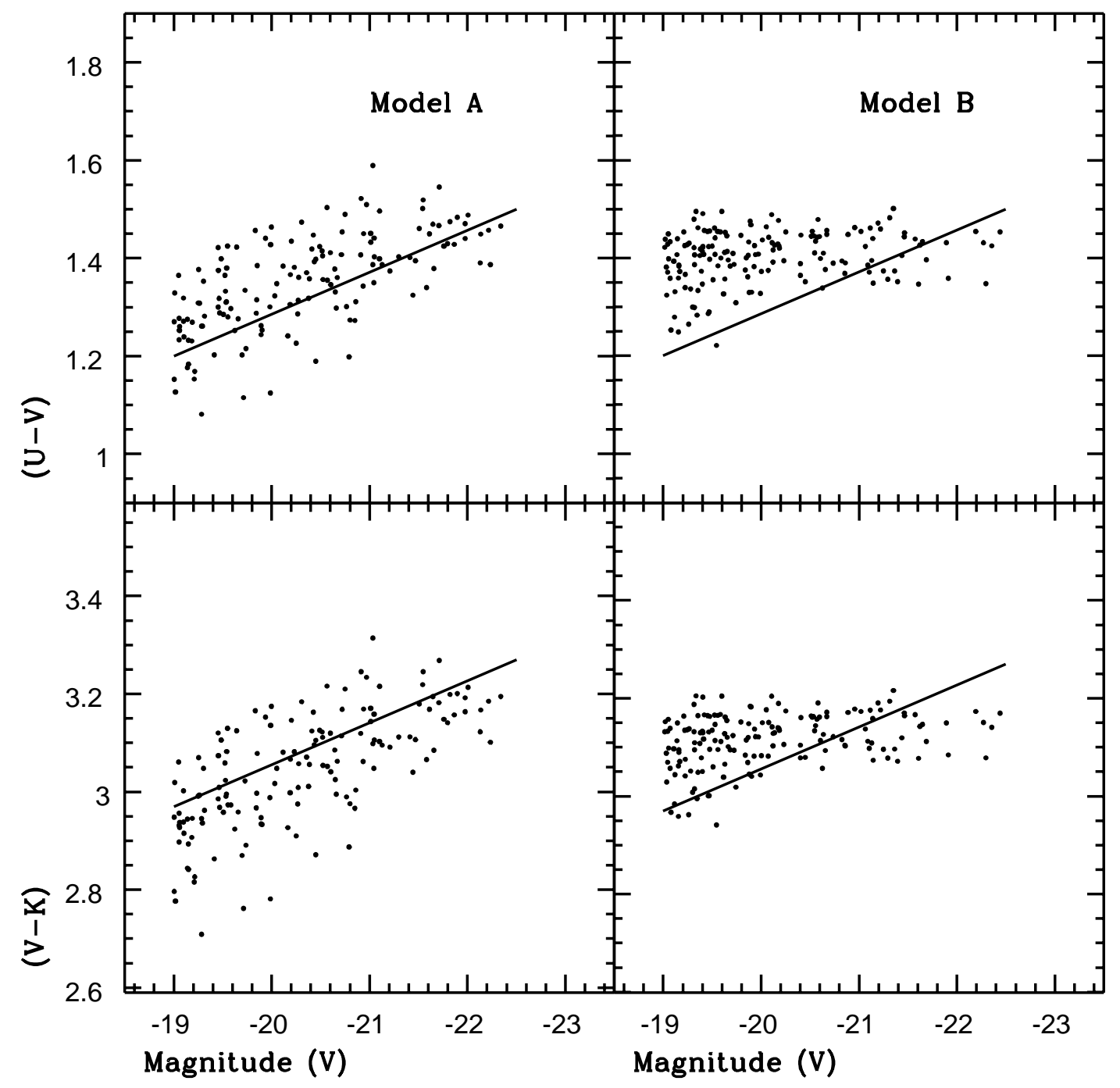

Figure 4: The $U-V$ and $V-K$ colour-magnitude relations of ellipticals galaxies in clusters of circular velocity $1000 \mathrm{~km} \mathrm{~s}^{-1}$. The solid lines are the fits to the data on Coma and Virgo ellipticals taken from Bower, Lucey \& Ellis (1992). 
section 3, are global indices, i.e. they are averaged over the entire stellar mass of the galaxy. For nearby ellipticals, however, the observed indices come from measurements in the central regions of the galaxies. Since the abundance of magnesium is known to fall off quite strongly with radius, we are forced to correct for this effect if we want to make a meaningful comparison with the data. We have used the observed $\mathrm{Mg}_{2}$-radius relation derived by Davies, Sadler \& Peletier (1993) $\left(\mathrm{Mg}_{2} \propto-0.059 \log \left(R / R_{e}\right), R_{e}\right.$ is the effective radius of the elliptical), to transform the global indices to central values. Since our model does not make any prediction for the sizes of elliptical galaxies, we use the observed relation between $V$-luminosity and effective radius given in Guzman, Lucey \& Bower (1993) to assign a value of $R_{e}$ to each of the model galaxies.

The uncorrected and corrected $\mathrm{Mg}_{2}-\sigma$ relations are shown in figure 5. The solid line is a fit to the data of Jørgensen, Franx \& Kjaergaard (1996). These authors quote $\mathrm{Mg}_{2}$ indices corrected to a circular aperture with diameter $1.19 \mathrm{~h}^{-1} \mathrm{kpc}$, equivalent to 3.4 arcsec at the distance of the Coma cluster. Since there is substantial scatter both in the observed $\mathrm{Mg}_{2}$ abundance gradients of ellipticals and in the values of $R_{e}$ at fixed luminosity, which we ignore in making the corrections, we will not attempt to analyze the scatter in the relation. What is clear, however, is that the slope of the corrected relation matches the observations rather well. To fit the observations, we require $V_{c} / \sigma \sim 2$.

In figure 6 , we plot the $M_{B}-\sigma$ Faber-Jackson relation for ellipticals (also assuming $V_{c} / \sigma=$ 2 ). The line is a fit to the $M_{B}-\sigma$ relation of Coma and Virgo cluster ellipticals from Dressler et al (1987). It is reassuring that we are able to fit both the $M_{B}-\sigma$ and the $\mathrm{Mg}_{2}-\sigma$ relation

with the same parameters. This gives us confidence that the different outputs of the spectral synthesis models are giving consistent results.

\section{The mass-to-light ratios of cluster ellipticals}

If the luminosity profiles and the dynamical structure of all elliptical galaxies are similar, the virial theorem implies that the mass-to-light ratio $M / L$ is a simple function of the effective radius $R_{e}$, the mean surface brightness $\langle I\rangle_{e}$, and the central velocity dispersion $\sigma$. All three quantities may be easily determined observationally.

It should be noted that the $M / L$ ratio is a measure of both the stellar population and the dark matter content of galaxies. Our models can be used to predict the stellar $M / L$ ratios of ellipticals. In figure 7, we show $(M / L)_{V}$ and $(M / L)_{K}$ as a function of stellar mass. The lines are fits to recent data on Coma cluster ellipticals taken from Mobasher et al (1997). It should be noted that the values we obtain for our stellar $M / L$ ratios are very sensitive to the choice of IMF. The results shown in figure 6 are for a Scalo (1986) IMF with a lower-mass cutoff of $0.1 M_{\odot}$. A Salpeter IMF with the same lower cutoff gives $M / L$ ratios a factor of $2-3$ higher. The slope of the relation is, however, independent of the IMF. As can be seen, our models fail to reproduce the observed increase in $M / L$ with mass. Our $(M / L)_{K}$ ratios actually decrease with mass, whereas Mobasher et al find $(M / L)_{K} \propto M^{\alpha}$, with $\alpha=0.14 \pm 0.01$.

One way of resolving this problem would be for more massive ellipticals to contain a larger fraction of dark matter than less massive ellipticals. In our model, this requires that more effective mixing of dark matter with stars take place during the formation of more massive ellipticals. As can be inferred from figure 2, massive ellipticals have typically more merging 

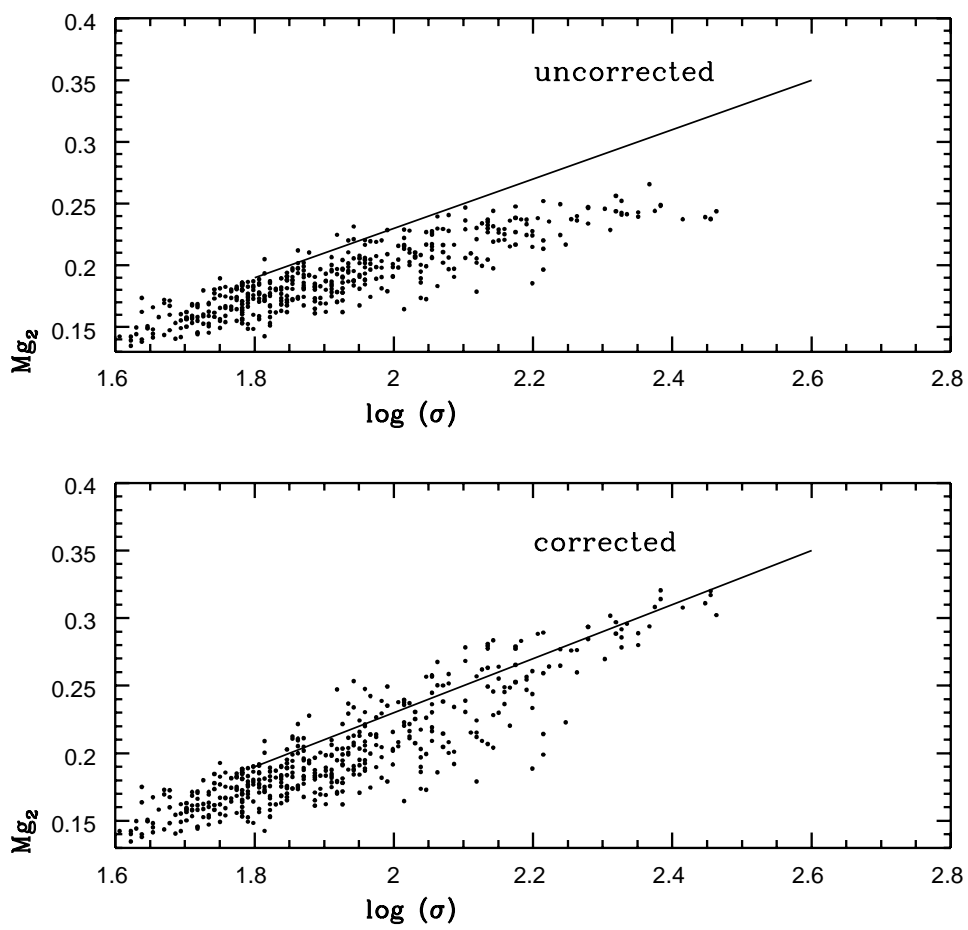

Figure 5: The corrected and uncorrected $\mathrm{Mg}_{2}-\sigma$ relations computed from our models. In the lower panel, the $\mathrm{Mg}_{2}$ indices have been transformed from global indices to central indices as described in the text. The line is a fit to the data from Jørgensen, Franx \& Kjaergaard (1995). 


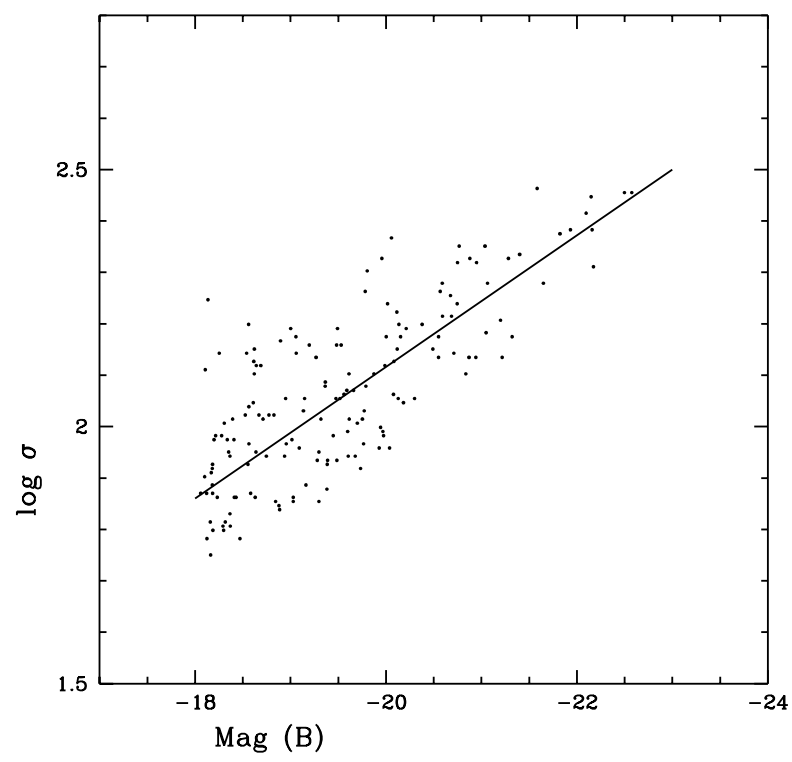

Figure 6: The B-band "Faber-Jackson" relation for ellipticals in the model. The line is a fit to the data for Coma Cluster ellipticals taken from Dressler et al (1987).

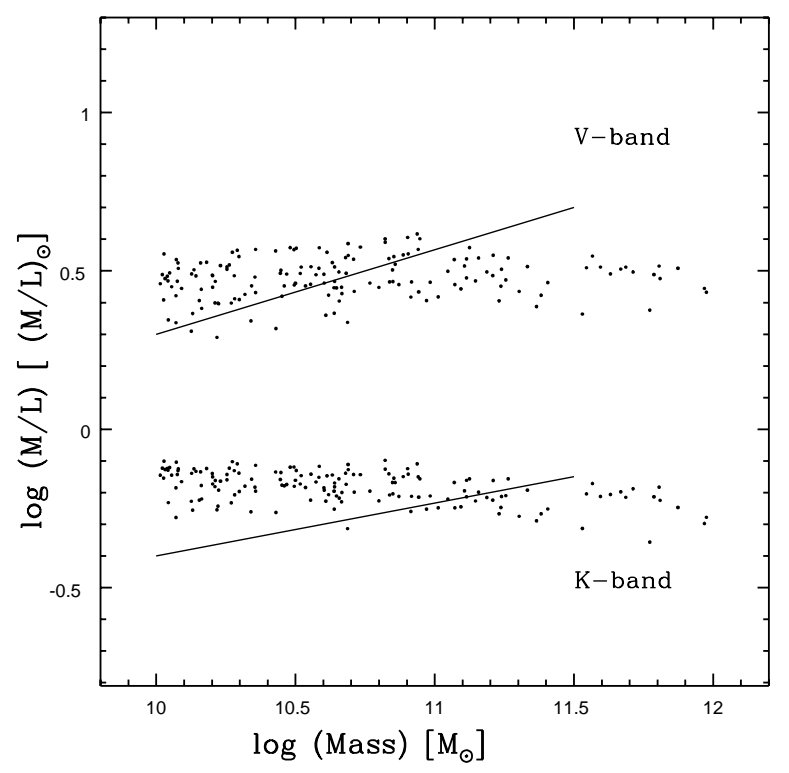

Figure 7: The $V$-band and $K$-band mass-to-light ratios of elliptical galaxies in clusters are plotted as a function of their stellar mass. The lines are fits to data from Mobasher et al (1997). 
events than less massive objects, so this appears plausible. Observationally, evidence for dark halos around ellipticals has been found through the studies of HI kinematics (e.g. Franx et al 1994), X-ray emission (e.g. Forman et al 1994), radial velocities of planetary nebulae and globular clusters (e.g. Mould et al 1990, Hui et al 1994), gravitational lensing (e.g. Maoz \& Rix 1993) and measurements of the shape of the stellar line-of sight velocity distribution (e.g. Carollo et al 1995). More recently, Rix et al (1997) have analyzed the velocity profile of the elliptical galaxy NGC 2434 and show that roughly half the mass within an effective radius is dark.

It should also be noted that recent analyses of the light profiles of elliptical galaxies show a slight breaking of the generally assumed homology. Allowing for this effect can substantially reduce the tilt of the $M / L$ relation (Hjorth \& Madsen 1996; Graham \& Colless 1997). This would also bring our results more in line with the data, and the variation in merger number with luminosity might offer an explanation for the variation in profile shape.

Finally, Chiosi et al (1997) claim to obtain a good fit to the observed $M / L$ relation using an IMF that varies as a function of the temperature, density and velocity dispersion of the gas that forms the elliptical. Their model does not attempt to place elliptical formation in its cosmological context.

\section{$7 \quad$ Evolution at high redshift}

In figure 8, we show age-luminosity and age-metallicity relations for elliptical galaxies in clusters of circular velocity $1000 \mathrm{~km} \mathrm{~s}^{-1}$ at $z=0.4$ and $z=1.5$. As can be seen, the metallicityluminosity relation does not evolve very much with redshift. By $z=1.5$, it has somewhat larger scatter, but an $M_{V}=-21$ elliptical still has a metallicity of 0.6 solar on average, compared with 0.75 solar at the present day. The age-luminosity relation shifts downwards with redshift, and the magnitude of the shift is roughly $t_{0}-t(z)$, where $t(z)$ is the age of the Universe at redshift $z$. Note that the scatter in age is smaller at high redshift, reflecting the compression of the time interval over which the stars in the elliptical are constrained to form. Thus in our models, elliptical galaxies of a given mass in high redshift clusters form and are enriched in much the same way as elliptical galaxies of the same mass in clusters at the present day. By selecting ellipticals in the richest clusters, one is automatically biasing ones sample of galaxies to the objects that turned around, collapsed and merged at the very highest redshifts. It is for this reason that the properties of these galaxies appear to follow the so-called "passive evolution" prediction (e.g. Aragon-Salamanca et al 1993; Stanford et al 1995; Dickinson 1995; Franx \& van Dokkum 1996; Bender, Ziegler \& Bruzual 1996; Ellis et al 1996). In practice, however, the ellipticals in the high $z$ clusters are not necessarily the direct progenitors of the Es in present-day clusters, so it is incorrect to set constraints on the formation of the present-day elliptical galaxy population using these objects (Charlot \& Silk 1994). This can only be done using a complete redshift survey of these objects (Kauffmann, Charlot \& White 1996).

In figure 9 we show the predicted restframe $U-V$ colour-magnitude relation at four different redshifts. For reference, the solid line is the observed relation of BLE at $z=0$. The dashed line indicates the shift in colour expected for a passively evolving population of solar metallicity with a formation redshift of 10 . Note that there is a progressive flattening 


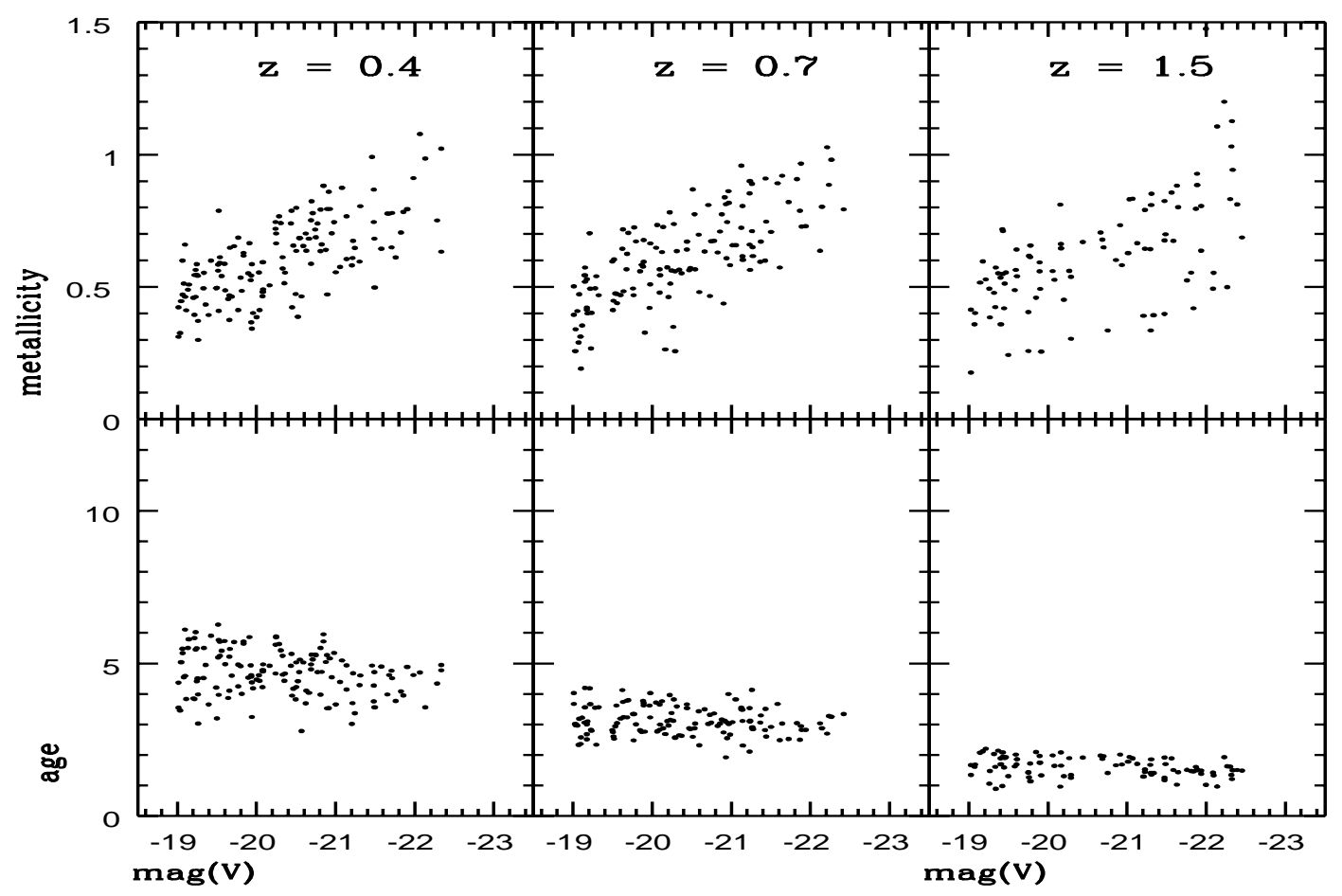

Figure 8: The metallicity-luminosity and age-luminosity relations of elliptical galaxies in clusters of circular velocity $1000 \mathrm{~km} \mathrm{~s}^{-1}$ at $z=0.4, z=0.7$ and $z=1.5$ 
in the slope of the colour-magnitude relation with redshift, although it only becomes really noticeable at $z>1$. This is caused by the fact that the bigger ellipticals have slightly younger mean ages in our model, and the effect of this on observables such as colour and magnitude, is much more pronounced at early times. The $\mathrm{Mg}_{2}-\sigma$ relation also shows a flattening at $z>1$, although it is a smaller effect than in the $U-V$ colours. The slope and scatter of the rest-frame $U-V$ colour-magnitude relation at each redshift are listed in Table 2 . The scatter in the rest-frame $U-V$ colours of ellipticals in clusters at $z \sim 0.54$ has been analyzed in detail by Ellis et al (1997). They find no change with respect to the $z=0$ relation, which is in good agreement with our results.

\section{Metals in the intracluster gas}

Since our model follows the exchange of metals between the stars, cold gas and hot gas component of galaxies, we are in a position to study the origin of metals in the intracluster medium. Recall that we chose the parameter $f$ to obtain a mean metallicity of 0.3 solar in the hot gas in a halo with circular velocity $1000 \mathrm{~km} \mathrm{~s}^{-1}$. Rather than the mass fraction of metals, many analyses have focused on the mass-to-light ratio of heavy elements in the ICM, as this quantity is independent of the degree to which the metals have been mixed with primordial gas. Most studies concentrate on iron, as this is the only element for which it has been possible, until recently, to derive accurate abundances using X-ray spectroscopy. For example, Renzini et al (1995) define the iron mass-to-light ratio (IMLR) as the ratio of the iron mass in the ICM to the total $B$-band light of the cluster. They derive values of $M_{F e}^{I C M} / L_{B}$ in the range $0.01-0.02 M_{\odot} / L_{\odot}$. The clusters we have modelled, which have mass $5 \times 10^{14} M_{\odot}$ and a baryon fraction of $10 \%$, typically contain a total $B$-band luminosity of $2 \times 10^{12} L_{\odot}$, of which $60-70 \%$ is in early-type galaxies. Our total $B$-luminosity and E/S0 fraction agree well with the values measured for the Virgo cluster (Kraan-Korteweg 1981). We obtain $M_{F e}^{I C M} / L_{B}=0.015 M_{\odot} / L_{\odot}$, in good agreement with the observations. Recall that our model has a yield of twice solar. This is larger than the conventional value adopted in most analyses (eg Arnaud et al 1992). However, as discussed by Renzini et al, the uncertainties in theoretical estimates of the yield of nucleosynthesis products from supernova explosions are rather large. Recent calculations by Tsujimoto et al (1995) actually give a (Sn II+Sn Ia) Fe yield close to twice solar, assuming a Salpeter IMF and the Sn Ia rate that best reproduces the abundance pattern of heavy elements in the solar neighborhood. We note that, according to Loewenstein \& Mushotsky (1996), the abundances of elements such as O, Ne, Si and S in nearby clusters may be even higher than that of $\mathrm{Fe}$, although the errors in the measurements are still quite large. The theoretical interpretation of these elements is in principle much simpler than that of Fe, because they are produced almost exclusively in Sn II explosions. Matching these results might require yields even higher than twice solar and would most likely only be compatible with a top-heavy IMF. We leave a more detailed examination of these issues to future work.

We can also use our models to study the history of the enrichment of the ICM and the distribution in mass (or circular velocity) of the galaxies that ejected the metals. This is illustrated in figure 10, where we plot the fraction of the total metals present in the present ICM that was ejected at a given redshift and from a galaxy of given circular velocity. As 


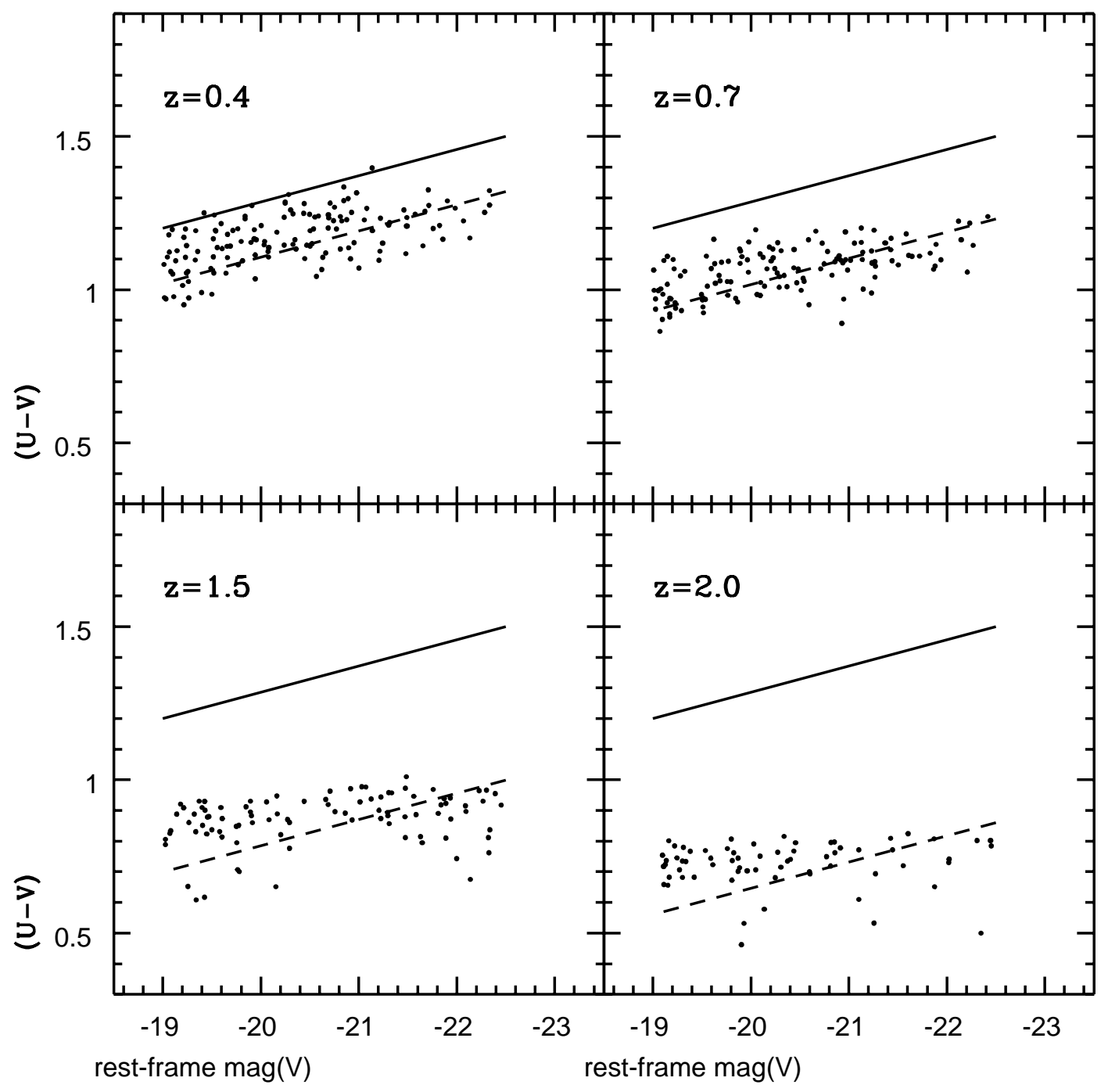

Figure 9: The rest-frame $U-V$ colour-magnitude relations of elliptical galaxies in clusters of circular velocity $1000 \mathrm{~km} \mathrm{~s}^{-1}$ at $z=0.4,0.7,1.5$ and 2.0. The solid lines are the $z=0$ data from Bower, Lucey \& Ellis (1992). The dashed lines show the shift in colour expected for a passively evolving population with $z_{\text {form }}=10$. 

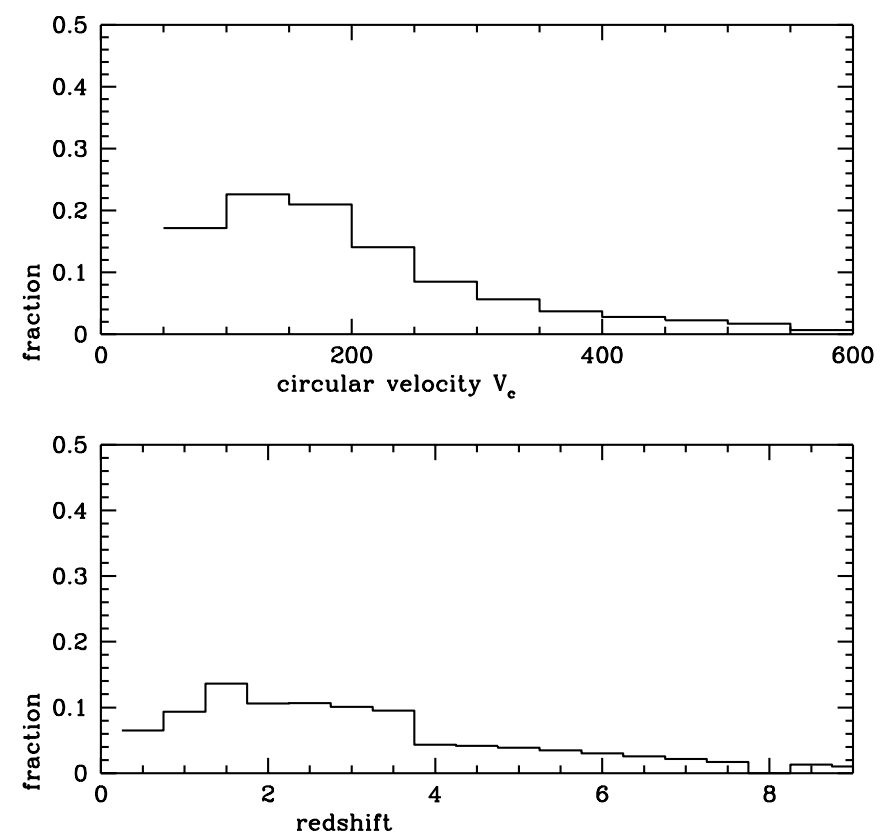

Figure 10: Top panel: the fraction of metals in the intracluster medium of a present-day cluster with circular velocity $1000 \mathrm{~km} \mathrm{~s}^{-1}$ ejected by disk galaxies with circular velocity $V_{c}$. Bottom panel: the fraction of metals in the ICM today that were ejected at reshift $z$.

can be seen, more than $80 \%$ of the metals were ejected at redshifts greater than 1, $60 \%$ at redshifts greater than 2 and $40 \%$ at redshifts greater than 3. More than $40 \%$ of the ejection was done by galaxies with circular velocities less than $125 \mathrm{~km} \mathrm{~s}^{-1}$, i.e. "dwarf" galaxies have contributed significantly to the metals in the ICM. Because the enrichment predominantly occurred at high redshift, our models predict that abundances in the ICM should evolve very little with redshift. Indeed, by $z=1.5$, the model predicts that the ICM metallicity is still $\sim 0.25$ solar. Our results are in good agreement with the lack of evolution seen in the Fe abundances of clusters out to $z \sim 0.3$ as measured by the ASCA satellite (Mushotsky \& Loewenstein 1997), although the model abundances show significantly less scatter than the data, suggesting that star formation in real cluster galaxies may be more stochastic than we have assumed.

\section{Discussion}

The failure to match the slope of the colour-magnitude relation of elliptical galaxies has been a serious defect of all previous attempts to model the formation of galaxies within the framework of a hierarchical cosmology (Lacey et al 1992 ; Kauffmann 1996b; Baugh, Cole \& Frenk 1996). We have demonstrated that the inclusion of chemical evolution in the model solves this problem. With a physically realistic choice of parameters we now obtain good fits to the $U-V$ and $V-K$ colour-magnitude relations as well as the $\mathrm{Mg}_{2}-\sigma$ relation. It is 
interesting that the colour-magnitude relation only extends to the brightest ellipticals if we require that feedback be very efficient, even in relatively massive galaxies. Using the value of $\epsilon$ given in Table 1 for model A, we can rewrite the cold gas reheating rate in equation 2 as

$$
\frac{d M_{\text {reheat }}}{d t}=\left(\frac{280 k m s^{-1}}{V_{c}}\right)^{2} \frac{d M_{*}}{d t}
$$

This implies that the reheating rate is $60 \%$ larger than the star formation rate for our own Milky Way. Because of the efficient feedback, our best fit model also requires a high value of $\Omega_{b}$, so that enough gas is able to cool and form the stars of the brightest galaxies.

Note that we have studied only global colours and metallicity indices values in this paper. It is well-known that elliptical galaxies exhibit strong gradients in their observed properties. In particular the nuclei of ellipticals host stellar populations with high metallicity $(\sim 0.5$ to $\sim 3 \mathrm{Z}_{\odot}$ ), a magnesium overabundance with respect to iron, and a small spread in metallicity. As discussed by Greggio (1997), the first two characteristics of the nuclei can be explained if gas is processed through multiple generations of stars on a short timescale. The small metallicity spread arises if the gas turning into stars in the nuclei has been pre-enriched. In N-body plus hydrodynamics simulations of the merger of two disk galaxies, gas is seen to be driven very effectively to the centre of the merger remnant, where it will likely turn into stars on a short timescale (Barnes \& Hernquist 1996). Whether this picture can account for the observed properties of elliptical nuclei has yet to be investigated in detail. In principle, it would be an interesting test of our models, which are able to predict when the mergers occurred, and the metallicity and mass of the gas in the disk-galaxy progenitors.

As in many other analyses, we fail to account for the tilt of the $M / L$ relation, if $M$ is purely a measure of the stellar mass of the galaxies (Djorgovski \& Santiago 1993; Worthey 1994). As we have discussed, there are several possible solutions to this problem, but one interesting one would be if more massive ellipticals contained a greater percentage of dark matter in their inner regions than less massive ellipticals. This might occur if dark matter is more efficiently mixed with the stars during the formation of the more massive systems. Clearly this is a problem that needs to be addressed using N-body simulations that take into account the full dynamics of the problem.

We have also predicted that the mass-metallicity relation for elliptical galaxies in clusters remains virtually unchanged out to high redshift. It is only the age of the galaxies that ought to decrease. This is a prediction that might be testable using stellar population indicators that are able to disentangle age and metallicity effects (eg Jones \& Worthey 1995). In addition, we predict that there should be a slight flattening in the slope of the colour-magnitude and $\mathrm{Mg}_{2}-\sigma$ relations at redshifts greater than 1, reflecting the fact that more massive ellipticals are slightly younger in age than the less massive ones.

Finally, X-ray satellites, such as ASCA, are beginning to provide a wealth of information about the abundances many different elements in the intracluster gas, as well as about the evolution in ICM abundances to high redshift (e.g. Mushotsky \& Loewenstein 1997). This information will provide further constraints on the models, in particular on our prescriptions for star formation and supernova feedback. These are indeed the most uncertain elements of our galaxy formation scheme. 


\section{Acknowledgments}

We thank Gustavo Bruzual, Marijn Franx, Bodo Ziegler and Alvio Renzini for helpful discussions. This work was carried out under the auspices of EARA, a European Association for

Research in Astronomy, and the TMR Network on Galaxy Formation and Evolution funded by the European Commission. 
Table 1: The parameters for the models

$\begin{array}{cccccc} & \Omega_{b} & \alpha & \epsilon & y & f \\ \text { Model A } & 0.1 & 0.2 & 0.4 & 2.0 & 0.3 \\ \text { Model B } & 0.06 & 0.1 & 0.05 & 1.2 & 0.45\end{array}$

Table 2: Best-fit regression parameters

$\begin{array}{lcc} & \text { slope } & \text { Std. Dev. } \\ \text { BLE }(U-V) & -0.076 & 0.037 \\ \text { BLE }(V-K) & -0.080 & 0.048 \\ \text { Model A }(U-V) z=0 & -0.067 & 0.019 \\ \text { Model A }(V-K) z=0 & -0.115 & 0.035 \\ \text { Model A }(U-V) z=0.4 & -0.057 & 0.016 \\ \text { Model A }(U-V) z=0.7 & -0.052 & 0.015 \\ \text { Model A }(U-V) z=1.5 & -0.038 & 0.017 \\ \text { Model A }(U-V) z=2.0 & -0.031 & 0.021\end{array}$

"Standard deviation" is calculated using the Median Absolute Difference technique, as in BLE 


\section{References}

Allard, F., \& Hauschildt, P.H. 1995, ApJ, 445, 433

Alongi, M., Bertelli, G., Bressan, A., Chiosi, C., Fagotto, F., Greggio, L., \& Nasi, E. 1993, A\&AS, 97,851

Aragón-Salamanca, A., Ellis, R.S., Couch, W.J. \& Carter, D., 1993, MNRAS, 262, 764

Arimoto, N. \& Yoshii, Y., 1987, A\&A, 173, 23

Arnaud, M., Rothenflug, R., Boulade, O., Vigroux, L. \& Vangioni-Flam, E., 1992, A\&A, 254, 49

Barnes, J.E. \& Hernquist, L., 1996, ApJ, 471, 115

Baugh, C.M., Cole, S. \& Frenk, C.S., 1996, MNRAS, 283, 1361

Bender, R., Burstein, D., \& Faber, S.M. 1993, ApJ, 411, 153

Bender, R., Ziegler, B. \& Bruzual, B., 1996, ApJ, 463, L51

Bessell, M.S., Brett, J., Scholtz, M., \& Wood, P. 1989, A\&AS, 77, 1

- 1991, A\&AS, 89, 335

Binney, J. \& Tremaine, S., 1987, Galactic Dynamics, Princeton University Press

Bond, J.R., Cole, S., Efstathiou, G. \& Kaiser, N. 1991, ApJ, 379, 440

Bower, R. 1991, MNRAS, 248, 332

Bower, R., Lucey, J.R. \& Ellis, R.S. 1992, MNRAS, 254, 601 (BLE)

Bressan, A., Chiosi, C. \& Tantalo, R., 1996, A\&A, 311, 425

Bressan, A., Fagotto, F., Bertelli, G., \& Chiosi, C. 1993, A\&AS, 100, 647

Bruzual A., G., Barbuy, B., Ortolani, S., Bica, E., Cuisinier, F., \& Schiavon, R. 1997, AJ, submitted

Carollo, C.M., de Zeeuw, P.T., van der Marel, R.P., Danziger, I.J. \& Qian, E.E., 1995, ApJ, 441, L25

Charlot, S., \& Silk, J. 1994, ApJ, 432, 453

Charlot, S., Worthey, G., \& Bressan, A. 1996, ApJ, 457, 625

Chiosi, C., Bressan, A., Portinaru, L., \& Tantalo, R. 1996, A\&A, submitted

Cole, S., Aragón-Salamanca, A., Frenk, C.S., Navarro, J.F. \& Zepf, S.E., 1994, MNRAS, 271, 781

Davies, R.L., Sadler, E. \& Peletier, R., 1993, MNRAS, 262, 650

Dickinson, M., 1996, in eds Buzzoni, A., Renzini, A. \& Serrano, A., eds, Fresh Views on Elliptical Galaxies, ASP Conf. Series Vol. 86, p283 
Djorgovski, S. \& Santiago, B.X., 1993, in Danziger, J. et al., eds, Proc. of the ESO/EIPC Workshop on Structure Dynamics and Chemical Evolution of Elliptical Galaxies, ESO Publication $45, \mathrm{p} 59$

van Dokkum, P.G. \& Franx, M, 1996, MNRAS, 281, 985

Dressler, A., 1984, ApJ, 286, 97

Dressler, A., Lynden-Bell, D., Burstein, D., Davies, R.L., Faber, S.M., Terlevich, R.J. \& Wegner, G., 1987, ApJ, 313, 42

Ellis, R.S., Smail, I., Dressler. A., Couch, W.J, 1996, preprint, astro-ph/9607154

Faber, S.M., 1977, in Tinsley, B.M. \& Larson, R.B., eds, The Evolution of Galaxies and Stellar populations, (Yale Observatory, New Haven), p157

Fagotto, F., Bressan, A., Bertelli, G., \& Chiosi, C. 1994a, A\&AS, 100, 647

- 1994b, A\&AS, 104, 365

- 1994c, A\&AS, 105, 29

Fluks, M. et al. 1994, A\&AS, 105, 311

Forman, W., Jones, C. \& Tucker, W., 1994, ApJ, 429, 77

Franx, M., van Gorkom, J. \& de Zeeuw, P.T., 1194, ApJ, 334, 613

Girardi, L., Bressan, A., Chiosi, C., Bertelli, G., \& Nasi, E. 1996, A\&AS, 117, 113

Graham, A. \& Colless, M., 1997, MNRAS, submitted

Greggio, L. 1997, MNRAS 285, 151

Gunn, J.E., 1982, in Bruck, H.A., Coyne, G.V. \& Longair, M.S., eds, Astrophysical Cosmology, (Vatican City: Potificia Academia Scientarium), 233

Guzman, R., Lucey, J.R. \& Bower, R.G., 1993, MNRAS, 265, 731 Hjorth, J. \& Madsen, J., 1995, ApJ, 445, 55

Hui, X., Ford, H.C., Freeman, K.C. \& Dopita, M.A., 1995, ApJ, 449, 592

Iglesias, C.A., Rogers, F.J., \& Wilson, B.G. 1992, ApJ, 397, 717

Jones, L.A., \& Worthey, G. 1995, ApJ, 446, L31

Jørgensen, I., Franx, M. \& Kjaergaard, P., 1996, MNRAS, 280, 167

Kauffmann, G., 1996a, MNRAS, 281, 475

Kauffmann, G., 1996b, MNRAS, 281, 487

Kauffmann, G. \& White, S.D.M. 1993, MNRAS, 261, 921

Kauffmann, G., White, S.D.M. \& Guiderdoni, B. 1993, MNRAS, 264, 201 (KWG) 
Kauffmann, G., Charlot, S. \& White, S.D.M., 1996, MNRAS, 283, L117

Kennicutt, R.C. 1983, ApJ, 272, 54

Kodama, T. \& Arimoto, N., 1997, A\&A, in press

Kraan-Korteweg, R.C., 1981, A\&A, 104, 280

Kurucz, R.L. 1992, in IAU Symp. 149, The Stellar Populations of Galaxies, ed. B. Barbuy \& A. Renzini (Dordrecht: Kluwer), 225

Lacey, C., Guiderdono, B., Rocca-Volmerange, B. \& Silk, J., 1993, ApJ, 402, 15

Larson, R.B., 1974, MNRAS, 166, 585

Lejeune, T., Cuisinier, F., \& Buser, R. 1997a, A\&A, in press

Loewenstein, M. \& Mushotsky, R.F., 1996, ApJ, 466, 695

Maoz, D. \& Rix, H.-W., 1993, ApJ, 416, 215

Matteuci, F. \& Tornambé,F., 1987, A\&A, 185, 51

Mobasher, B., Guzman, R., Aragón-Salamanca, A. \& Zepf, S., 1997, MNRAS, submitted

Mould, J.R., Oke, J.B., de Zeeuw, P.T. \& Nemec, J.M., 1990, AJ, 99, 1823

Mushotsky, R.F. \& Loewenstein, M., 1997, preprint, astro-ph/9702149

Renzini, A., Ciotti, L., Dercole, A. \& Pellegrini, S., 1993, ApJ, 419, 52

Rix, H.-W., de Zeeuw, P.T., Carollo, C.M., Cretton, N. \& van der Marel, M. 1997, preprint, astro-ph/9702126

Roberts, M.S. \& Haynes, M.P., 1994, ARA\&A, 32, 115

Scalo, J.N., 1986, Fundamentals of Cosmic Physics, Vol. 11, p1

Stanford, S.A., Eisenhardt, P. \& Dickinson, M., 1995, ApJ, 450, 512

Tinsley, B.M., 1980, Fundamentals of Cosmic Physics, Vol. 5, p287

Tsujimoto, T., Nomoto, K., Yoshii, Y., Hashimoto, M., Yanagida, S. \& Thielemann, F.-K., 1995, MNRAS 277, 945

Spitzer, L., 1990, ARA\&A, 28, 71

Turner, M.S., 1996, preprint, astro-ph/9610158

Vader, J.P., 1986, ApJ, 305, 669

White, D.A. \& Fabian, A.C., 1995, MNRAS, 273, 72

White, S.D.M. \& Frenk, C.S., 1991, ApJ, 379, 52 
White, S.D.M., Navarro, J.F., Evrard, A.E. \& Frenk, C.S., 1993, Nature, 366, 429

Worthey, G., 1994, ApJS, 95, 107

Worthey, G., Faber, S.M., \& Gonzalez, J.J. 1992, ApJ, 398, 69

Worthey, G., Faber, S.M., Gonzalez, J.J., Burstein, D. 1994, ApJS, 94, 687

Worthey, G., 1996, in eds Buzzoni, A., Renzini, A. \& Serrano, A., eds, Fresh Views on Elliptical Galaxies, ASP Conf. Series Vol. 86, p203 